\title{
Genotipificación de los genes rgpA y kgp que codifican para las gingipaínas de Porphyromonas gingivalis
}

\author{
Genotyping of rgpA and kgp genes encoding Pophyromonas \\ gingivalis gingipains
}

\author{
Abusleme $\mathrm{L}^{1}$, Blanc $\mathrm{V}^{2}$, Léon $\mathrm{R}^{2}$, Gamonal $\mathrm{J}^{3}$, Silva $\mathrm{N}^{1}$
}

\begin{abstract}
RESUMEN
Porphyromonas gingivalis es un microorganismo fuertemente asociado con la etiología de la periodontitis. Esta bacteria posee varios factores de virulencia, dentro de los que destacan las gingipaínas, debido a sus múltiples acciones relacionadas con la destrucción de la matriz extracelular del tejido conectivo periodontal, la modulación del sistema inmune del hospedero y la estimulación de la expresión de citoquinas pro-inflamatorias. Estas proteinasas tienen afinidades específicas siendo Arg-gingipaínas (RgpA y RgpB, codificadas por los genes rgpA y rgpB, respectivamente) y Lys-gingipaínas (Kgp, codificada por el gen $\mathrm{kgp}$ ). Se ha descrito que existen polimorfismos en los genes que codifican para esta proteinasas. El objetivo del presente estudio fue describir la frecuencia de los genotipos identificados para los genes rgpA y kgp en aislados clínicos de $P$. gingivalis, obtenidos desde pacientes con periodontitis. Para ello se utilizó amplificación por PCR de los genes rgpA y kgp, seguido de análisis de restricción. De un total de 47 aislados provenientes de 4 individuos con periodontitis crónica y 2 con periodontitis agresiva, se genotipificaron 38 aislados para el gen $\operatorname{rgpA}$, exhibiendo la totalidad de éstos el patrón electroforético A (100\%). Para el gen kgp se genotipificaron 43 aislados, presentando 28 de ellos (65.2\%) el perfil electroforético $\mathrm{kgp}-\mathrm{l}$ y 15 aislados (34.8\%) el perfil $\mathrm{kgp}$ - Il. En los aislados provenientes de un individuo fue posible apreciar ambos genotipos descritos para el gen $\mathrm{kgp}$. Los resultados indican un predominio del patrón electroforético A (rgpA) y que el genotipo kgp-l fue el más frecuentemente encontrado de los genotipos kgp.
\end{abstract}

Rev. Clin. Periodoncia Implantol. Rehabil. Oral Vol. 5(3); 136-139, 2012.

Palabras clave: Porphyromonas gingivalis, gingipaínas, genotipos, kgp, rgpA.

\begin{abstract}
Porphyromonas gingivalis is a microorganism strongly associated with the etiology of periodontitis. This periodontal bacterium possesses an array of virulence factors, among which gingipains have a key importance, being involved with extracellular matrix destruction of periodontal tissues, modulation of host immune response and stimulation in the production of pro-inflammatory cytokines by different types of cells. These proteinases have specific affinities, being Arg-gingipains (RgpA and RgpB, encoded by rgpA and rgpB genes, respectively) and Lys-gingipains (Kgp, encoded by the kgp gene). It has been described that there are polymorphisms in the genes encoding for gingipains. Therefore, the aim of the present study was to describe the frequency of $\operatorname{rgpA}$ and $\mathrm{kgp}$ genotypes in clinical isolates of $P$. gingivalis obtained from periodontitis patients. For determining the rgpA and $\mathrm{kgp}$ genotypes, we used PCR amplification and restriction analysis. From 47 isolates obtained from 4 individuals with chronic periodontitis and 2 subjects with aggressive periodontitis, 38 were typified for $\operatorname{rgpA}$ gene and all exhibited the electrophoretic pattern A (100\%). For kgp gene, we characterized 43 isolates, 28 of them (65.2\%) with the $\mathrm{kgp}-\mathrm{l}$ electrophoretic profile and 15 isolates (34.8\%) with the $\mathrm{kgp}-/ /$ profile. In the isolates belonging to one individual, we found both genotypes of $\mathrm{kgp}$ gene. The results indicate a clear predominance of the electrophoretic pattern A (for $\mathrm{rgpA}$ gene) and $\mathrm{kgp}-\mathrm{I}$ genotype was the most frequently found of the kgp genotypes.
\end{abstract}

Rev. Clin. Periodoncia Implantol. Rehabil. Oral Vol. 5(3); 136-139, 2012.

Key words: Porphyromonas gingivalis, gingipains, genotypes, kgp, rgpA.

\section{INTRODUCCIÓN}

Porphyromonas gingivalis es considerado un patógeno representativo del biofilm subgingival asociado a periodontitis crónica ${ }^{(1,2)}$ y se ha descrito como un marcador de progresión de esta patología ${ }^{(3,4)}$. Este microorganismo posee múltiples factores de virulencia, tales como: cápsula, fimbria, lipopolisacárido (LPS) y proteinasas, dentro estas últimas destacan un grupo de cisteína proteinasas, llamadas "gingipaínas"(5). Se han identificado dos tipos de gingipainas, definidas por la especificidad de sus sustratos, siendo éstas arginina-específicas (Arg-gingipainas o Rgp) y lisina-especificas (Lys- gingipainas o Kgp) ${ }^{(6)}$. A su vez, hay dos variantes de Arg-gingipainas, RgpA y RgpB, codificadas por los genes $r g p A$ (previamente prpR1) y $r g p B$, respectivamente. A nivel estructural, se diferencian en que RgpA posee un dominio catalítico y un dominio adhesina/hemaglutinina, en cambio, RgpB sólo presenta un dominio catalítico ${ }^{(7)}$. Respecto a las Lys-gingipainas, Kgp, al igual que RgpA, posee un dominio catalítico y otro adhesina/hemaglutinina, y es codificada por el gen $\mathrm{kgp}^{(8)}$. Estas proteinasas de $P$. gingivalis (RgpA, RgpB y Kgp), tienen un amplio espectro de acción, se ha determinado que participan directamente en la proteólisis de la matriz extracelular del tejido conectivo periodontal(9), en la obtención de hierro y hemina, esenciales para el crecimiento de $P$. gingivalis $^{(10)}$, y además, se ha determinado que ayudan en la adhesión de esta bacteria a células epiteliales $^{(11)}$ y fibroblastos ${ }^{(12)}$, favoreciendo la colonización de los tejidos periodontales. Por otra parte, recientemente se ha demostrado que estas enzimas tienen la capacidad de inducir apoptosis en células epiteliales, probablemente a través de la degradación de actina, lo cual conlleva a un colapso del citoesqueleto ${ }^{(13)}$. Adicionalmente, las gingpaínas

1. Laboratorio de Microbiología Oral. Departamento de Patología. Facultad de Odontología, Universidad de Chile. Santiago, Chile.

2. Laboratorio de Microbiología. Dentaid S.L. Barcelona, España.

3. Laboratorio de Biología Periodontal Departamento de Odontología Conservadora. Facultad de Odontología, Universidad de Chile. Santiago, Chile.

Correspondencia autor: Loreto Abusleme Ramos. loreabusleme@uchile.cl. Financiamiento: Proyecto FONDECYT 1090046 y Laboratorios Dentaid S.L. Barcelona, España. Trabajo recibido el 01/09/2012. Aprobado para su publicación el 24/10/2012. 
ejercen múltiples roles a nivel de la modulación del sistema inmune del hospedero, ya que son capaces de degradar defensinas ${ }^{(14)}$, varios componentes del complemento ${ }^{(15)}$ y receptores de células T (CD2, CD4 y CD8 $)^{(16)}$. También, se ha determinado que estimulan la expresión de citoquinas pro-inflamatorias en células epiteliales orales ${ }^{(17)}$ y fibroblastos gingivales ${ }^{(18)}$

Debido a las múltiples acciones descritas para las gingipainas a nivel de la de la virulencia de este microorganismo y su relación con la periodontitis, algunos autores se han centrado en el estudio del polimorfismo de los genes que codifican para estas proteinasas y su relación con la virulencia de las diferentes cepas de $P$. gingivalis. Allaker y cols. ${ }^{(19)}$, utilizando análisis de restricción, describieron 3 patrones electroforéticos (patrones $\mathrm{A}, \mathrm{B}$ y $\mathrm{C}$ ) para el gen $\operatorname{rgpA}$ y en otro estudio posterior Beikler y cols. ${ }^{(20)}$, a través de la misma metodología, identificaron 2 genotipos para el gen $\mathrm{kgp}$.

A la fecha, sólo un estudio ha explorado la prevalencia de ambos genotipos (rgpA y $k g p)^{(21)}$, por cuanto el objetivo de este trabajo fue describir la frecuencia de los genotipos identificados para los genes $\operatorname{rgpA}$ y kgp en aislados clínicos de $P$. gingivalis provenientes de sujetos chilenos con periodontitis.

\section{METODOLOGÍA}

\section{Sujetos, Procedimientos Microbiológicos y Extracción de DNA}

Los 47 aislados clínicos de $P$. gingivalis utilizados en este estudio se obtuvieron de acuerdo a lo descrito por Abusleme y cols. ${ }^{(22)}$. Brevemente, de los 6 individuos incluidos en dicho estudio, 4 fueron diagnosticados con Periodontitis Crónica y dos con Periodontitis Agresiva. Las muestras fueron obtenidas insertando dos conos de papel estériles en los sitios más profundos de cada cuadrante, siendo inmediatamente sembradas e incubadas en anaerobiosis durante 14 días. Posteriormente se procedió a la identificación fenotípica de $P$. gingivalis en los aislados de bacterias pigmentadas de negro, seguido de la extracción de DNA, su cuantificación e identificación molecular, de acuerdo a lo descrito en el estudio antes mencionado ${ }^{(22)}$.

\section{Amplificación de los Genes rgpA y kgp Mediante PCR}

\section{Amplificación del Gen rgpA}

Para determinar si existía polimorfismo en el gen $\operatorname{rgp} A$, se amplificó un fragmento de $1.7 \mathrm{~kb}$ correspondiente a la región $\alpha$ de del gen. Para la reacción de PCR se utilizaron los partidores descritos por Allaker y cols. ${ }^{(19)}$, cuyas secuencias fueron $F$ 5' AGT GAG CGA AAC TTC GGA GC 3' y R 5'- GGT ATC ACT GGG TAT AAC CTG TCC- 3'. El volumen final de cada reacción fue $50 \mu$, los cuales contenían $1 X$ de buffer de PCR, $2.5 \mathrm{mM}$ de $\mathrm{MgCl} 2,0.2 \mathrm{mM}$ de una mezcla equimolar de dNTP, $1 \mu \mathrm{M}$ de cada uno de los partidores, $1.5 \mathrm{U}$ de Taq DNA polimerasa (Roche Diagnostics $\mathrm{GmbH}$, Mannheim, Germany) y $200 \mathrm{ng}$ de DNA genómico de $P$. gingivalis. Como control positivo se utilizó DNA genómico de la cepa ATCC 33277 de $P$. gingivalis $(200 \mathrm{ng}$ ) y como control negativo se usó agua miliq. Las reacciones de amplificación se llevaron a cabo en un termociclador Gene Amp PCR system 2400 (Perkin Elmer Corporation, Foster, CA, USA), de acuerdo a las siguientes condiciones: una desnaturalización inicial a $95^{\circ} \mathrm{C}$ por 10 minutos, seguido de 30 ciclos de desnaturalización a $95^{\circ} \mathrm{C}$ por 1 minuto, alineamiento a $56^{\circ} \mathrm{C}$ por 1 minuto y extensión a $72^{\circ} \mathrm{C}$ por 1 minuto y 30 segundos, concluyendo con una extensión final a $72^{\circ} \mathrm{C}$ por 7 minutos. Para observar los productos de la reacción de PCR, se realizó una electroforesis en gel de agarosa al $1 \%$ y tinción con bromuro de etidio, siendo visualizados y registrados a través del sistema Gel-DocTM XR+ (Bio-Rad, Hércules, CA, USA). En las muestras que se observó el fragmento de $1.7 \mathrm{~kb}$, se realizó nuevamente una amplificación para poder purificar posteriormente una cantidad suficiente de producto de PCR.

\section{Amplificación del Gen kgp}

Mediante PCR se amplificó un fragmento de $890 \mathrm{bp}$, que codifica para el dominio catalítico de este gen. La reacción se realizó utilizando los partidores descritos por Beikler y cols. ${ }^{(20)}$, cuyas secuencias fueron F 5'- GAA CTG ACG AAC ATC ATT G - 3' y R 5'- GCT GGC ATT AGC AAC ACC TG- 3'. El volumen de reacción fue $50 \mu \mathrm{l}$, que contenían: Buffer de PCR 1X, $2.5 \mathrm{mM}$ de MgCl2, $0.2 \mathrm{mM}$ de una mezcla equimolar de dNTP, $1 \mu \mathrm{M}$ de cada uno de los partidores, $0.75 \mathrm{U}$ de Taq DNA polimerasa (Roche Diagnostics $\mathrm{GmbH}$, Mannheim, Germany) y 100 ng de DNA genómico de $P$. gingivalis. Como control positivo se utilizó DNA genómico de la cepa ATCC 33277 de $P$. gingivalis (100 ng) y como control negativo se usó agua miliq. Las reacciones de amplificación se realizaron en un termociclador Gene Amp PCR system 2400 (Perkin Elmer Corporation, Foster, CA, USA), de acuerdo a las siguientes condiciones: una desnaturalización inicial a $95^{\circ} \mathrm{C}$ por 10 minutos, seguido de 35 ciclos de desnaturalización a $95^{\circ} \mathrm{C}$ por 1 minuto, alineamiento a $56^{\circ} \mathrm{C}$ por 1 minuto y extensión a $72^{\circ} \mathrm{C}$ por 1 minuto y 30 segundos, concluyendo con una extensión final a $72^{\circ} \mathrm{C}$ por 7 minutos. Para visualizar los productos de la reacción de PCR, se realizaron los mismos procedimientos detallados para los amplicones del gen rgpA. En las muestras que se pudo observar el fragmento de $890 \mathrm{bp}$, se realizó nuevamente una amplificación para poder purificar una cantidad suficiente de producto de PCR.

\section{Purificación de los Productos de PCR}

Los productos de PCR fueron purificados a partir de un gel de agarosa LE (low electroendosmosis; Roche Diagnostics $\mathrm{GmbH}$ Mannheim, Germany) al 1\%, utilizando el kit Illustra GFX' ${ }^{\mathrm{TM}}$ PCR DNA and Gel Band Purification (GE Healthcare, Buckinghamshire, UK) siguiendo las instrucciones del fabricante.

\section{Análisis de Restricción Genes rgpA y kgp}

Los productos de PCR del gen rgpA purificados se digirieron utilizando la enzima de restricción Rsa I (Roche Diagnostics $\mathrm{GmbH}$ Mannheim, Germany) y los amplicones del gen kgp se digirieron con la enzima de restricción Mse I (Roche Diagnostics $\mathrm{GmbH}$, Mannheim, Germany). Los fragmentos de la digestión se del gen rgpA y kgp se observaron realizando electroforesis en geles de agarosa al $5 \%$ y al $2 \%$, respectivamente. Los geles fueron teñidos con bromuro de etidio y visualizados con luz ultravioleta utilizando el sistema Gel-DocTM XR+ (Bio-Rad, Hercules, CA, USA).

\section{RESULTADOS}

\section{Identificación de Genotipos del Gen rgpA}

De un total de 47 aislados identificados previamente como $P$. gingivalis, se genotipificaron 38 , en los restantes no se logró obtener un producto de PCR para realizar la digestión enzimática. Todos los aislados genotipificados $(100 \%)$ para el gen rgpA presentaron el mismo patrón electroforético, posterior a la restricción con Rsa I. Este patrón era similar al patrón electroforético A descrito por Allaker y cols. ${ }^{(19)}$. Como se observa en la Figura 1, de la digestión con la enzima Rsa I del amplicón de 1.7 $\mathrm{kb}$, se obtuvieron 7 fragmentos de 552, 543,171, 159, 117, 111 y 49 bp lo que corresponde al patrón $\mathrm{A}$. Cabe destacar que si bien no se alcanzó a visualizar el fragmento de $49 \mathrm{bp}$, su presencia se comprobó analizando la secuenciación del amplicón del gen rgpA (datos no mostrados).

\section{Identificación de Genotipos del Gen kgp}

Se genotipificaron 43 de los 47 aislados identificados previamente como $P$. gingivalis, dado que no fue posible obtener un producto de PCR para la digestión enzimática en 4 de ellos. De los 43 aislados genotipificados, $28(65.2 \%)$ presentaron el patrón electroforético kgp-l y 15 aislados (34.8\%) mostraron el patrón electroforético kgp-II, de acuerdo a la metodología descrita por Beikler y cols. ${ }^{(20)}$. En la Figura 2, se observa el perfil $\mathrm{kgp}-\mathrm{l}$ que se caracteriza por presentar 3 fragmentos de restricción de 447, 288 y 135 bp. Por otra parte, el patrón kgp-ll corresponde al fragmento de 890 bp ya que no posee sitios reconocidos por la enzima Mse I.

\section{Distribución y Frecuencia de Genotipos}

En la Tabla 1 se describe un resumen de la distribución de los genotipos caracterizados en este estudio, el número de veces que fueron detectados y el diagnóstico clínico de los pacientes de los cuáles fueron obtenidos los aislados. Como se señaló en la metodología, para el desarrollo este trabajo, se utilizaron los aislados clínicos provenientes de 
Ios pacientes que fueron reclutados para el estudio de Abusleme y cols. ${ }^{(22)}$, siendo éstos 6 pacientes, 4 con diagnóstico de periodontitis crónica y 2 con diagnóstico de periodontitis agresiva. Respecto a lo obtenido en la genotipificación del gen $\operatorname{rgp} A$, en los pacientes con periodontitis crónica y agresiva, se encontró el patrón electroforético $A \quad(n=38$, $100 \%$ ), y no se observó diferencias entre ambas patologías. Por otra parte, los genotipos caracterizados para el gen kgp (kgp-l y kgp-ll) fueron encontrados en los aislados provenientes de los dos grupos de pacientes, existiendo un predominio del perfil $\mathrm{kgp}-\mathrm{I}$ con un $65.2 \%$ de los aislados analizados. Cabe destacar que en los aislados de $P$. gingivalis provenientes de un mismo paciente (con diagnóstico clínico de periodontitis crónica), fue posible observar ambos perfiles electroforéticos para este gen (kgp-l y kgp-II).

Tabla 1. Resumen patrones electroforéticos genes $\operatorname{rgpA}$, kgp y el diagnóstico clínico de los pacientes estudiados.

\begin{tabular}{|c|c|c|c|}
\hline Diagnóstico Clínico & Paciente & Patrón rgpA & Patrón kgp \\
\hline \multirow{4}{*}{$\begin{array}{l}\text { Periodontitis crónica } \\
\qquad(n=4)\end{array}$} & 1 & $A(n=5)$ & kgp-I $(n=8)$ \\
\hline & 2 & $A(n=9)$ & $\operatorname{kgp}-I(n=10)$ \\
\hline & 3 & $A(n=5)$ & kgp- $-1(n=7)$ \\
\hline & 4 & $A(n=7)$ & $k g p-I(n=2)$ y $k g p-I I(n=3)$ \\
\hline \multirow{2}{*}{$\begin{array}{l}\text { Periodontitis agresiva } \\
\qquad(n=2)\end{array}$} & 5 & $A(n=7)$ & kgp-I $(n=8)$ \\
\hline & 6 & $A(n=5)$ & $\operatorname{kgp}-I I(n=5)$ \\
\hline Resumen & $n=6$ & $\begin{array}{l}38 \text { aislados patrón } \\
\qquad A(100 \%)\end{array}$ & $\begin{array}{l}\quad 43 \text { aislados } \\
\text { Patrón kgp-l = } 28(65.2 \%) \\
\text { Patrón kgp- } / l=15(34.8 \%)\end{array}$ \\
\hline
\end{tabular}

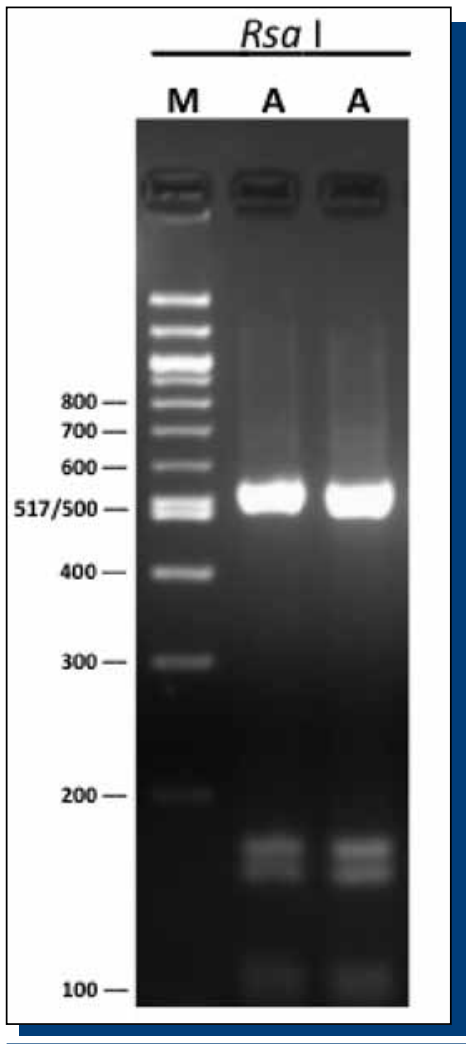

Figura 1. Detección de genotipos rgpA utilizando análisis de restricción digiriendo con la enzima Rsa I. A: patrón electroforético A proveniente de dos aislados clínicos de $P$. gingivalis ${ }^{(19)}$. M: marcador de peso molecular $100 \mathrm{bp}$.

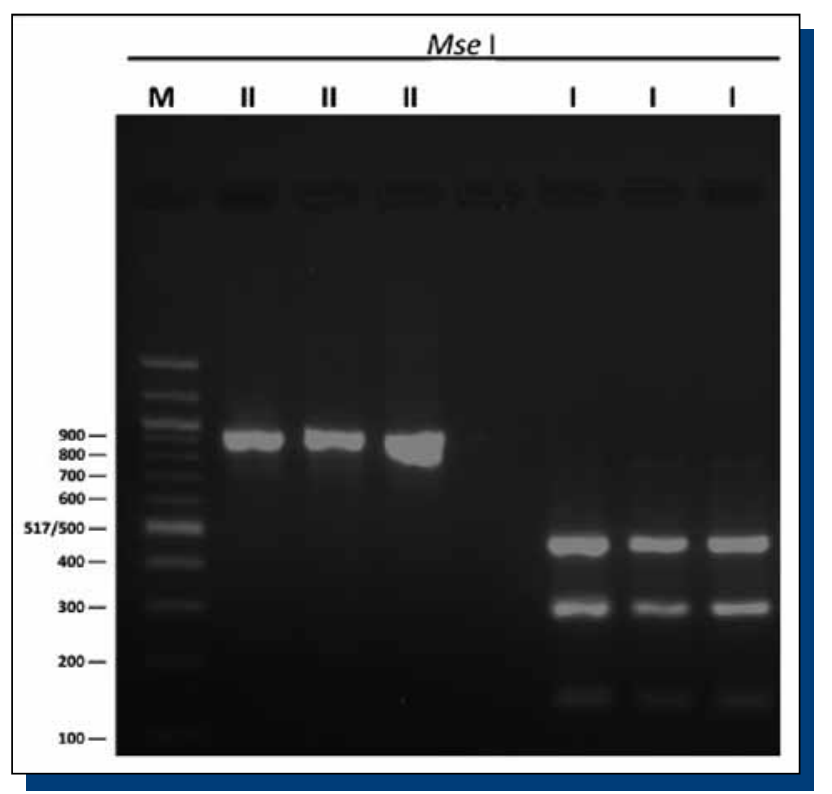

Figura 2. Detección de genotipos kgp utilizando análisis de restricción digiriendo con la enzima Mse I. I: patrón electroforético $\mathrm{kgp}-\mathrm{l}$, patrón electroforético $\mathrm{kgp}-/ /$ provenientes de 6 aislados clínicos de $P$. gingivalis. M: marcador de peso molecular $100 \mathrm{bp}$.

\section{DISCUSIÓN}

En el presente trabajo, se caracterizaron los genotipos rgpA y kgp provenientes de aislados clínicos de $P$. gingivalis, analizando 47 aislados obtenidos de 6 sujetos con diagnósticos de periodontitis crónica y agresiva ${ }^{(22)}$. La totalidad de los aislados que pudieron ser genotipificados presentaron el patrón electroforético $\mathrm{A}$, descrito para el gen $\operatorname{rgp} A$ y hubo un predominio del genotipo $\mathrm{kgp}-\mathrm{l}$, dentro de los polimorfismos descritos para el gen $k g p$.

Los datos presentados en este estudio concuerdan con lo obtenido por otros autores anteriormente. Analizando 43 aislados de $P$. gingivalis, Allaker y cols. ${ }^{(19)}$, describieron 3 patrones electroforéticos de restricción (A, B y C) al caracterizar los amplicones del gen rgpA. Dichos autores, encontraron que el patrón electroforético $A$ fue el más prevalente $(77 \%)$, seguido del patrón B $(21 \%)$ y el patrón C $(2 \%)$. Por otra parte, Yoshino y cols. ${ }^{(21)}$, evaluaron en 62 aislados de $P$. gingivalis la variabilidad en genes que codifican para factores de virulencia (fimA, $k g p$ y $\operatorname{rgpA}$ ) y sus serotipos capsulares. En relación a los genotipos descritos para el gen $\operatorname{rgp} A$, estos autores encontraron una prevalencia del $75.8 \%$ patrón electroforético $\mathrm{A}$, un $21 \%$ presentó el patrón $\mathrm{B}$ y un $3.2 \%$ correspondió al patrón $\mathrm{C}^{(21)}$. Los resultados anteriormente expuestos corresponden a los únicos trabajos de investigación en la literatura que utilizan esta metodología (análisis de restricción) para describir genotipos del gen rgpA. En el presente estudio, también hubo un predominio del patrón electroforético $\mathrm{A}$, aunque la prevalencia encontrada fue mayor dado que la totalidad de los aislados $(100 \%)$ correspondió a dicho perfil, no detectándose los patrones $\mathrm{B}$ y $\mathrm{C}$. Este resultado podría deberse en parte al número de aislados genotipificados que fue levemente inferior al revisado en los trabajos antes mencionados, por cuanto quizás no fue posible visualizar dichos perfiles (B y $C$ ). Sin embargo, los datos obtenidos coinciden claramente en la mayor frecuencia detectada del patrón electroforético A, lo cual concuerda con el análisis realizado en el mismo trabajo citado de Allaker y cols. ${ }^{(19)}$, quienes utilizando Southern blot hybridization, refuerzan el hecho de que el gen $\operatorname{rgp} A$ es altamente conservado. Del mismo modo, se ha descrito a través de la secuenciación de los genes $\operatorname{rgp} A$ y $\operatorname{rgp} B$, que el gen $\operatorname{rgp} A$ no presenta polimorfismo en la secuencia nucleotídica que codifica las regiones que determinan la especificidad del sustrato de $\operatorname{Rgp} A$ y $\operatorname{Rgp} B$, a diferencia del gen $\operatorname{rgp} B$ en donde fue posible describir 5 genotipos distintos de acuerdo a las variaciones de su secuencia ${ }^{(23)}$.

En relación a los genotipos kgp, se observó un predominio del perfil $k g p-I(65.2 \%)$ y el perfil $k g p-I /$ se detectó en un $34.8 \%$ en los aislados analizados, lo cual es similar a lo obtenido por Beikler y cols. ${ }^{(20)}$, quienes describieron que un $57.8 \%$ de los pacientes analizados presentaba el genotipo $\mathrm{kgp}-\mathrm{l}$, mientras que el genotipo $\mathrm{kgp}$-// fue caracterizado en el $42.2 \%$ de los sujetos incluidos en dicho estudio ${ }^{(20)}$. Además, los datos obtenidos 
también concordaron con lo encontrado por Yoshino y cols. ${ }^{(21)}$, en donde un $56.5 \%$ de los aislados revisados en dicho estudio presentaron el perfil $k g p-I$ y un $43.5 \%$ el perfil $k g p-I I$. La mayor prevalencia del perfil $k g p-I$, podría estar indicando que esos genotipos poseen una mejor capacidad de adaptación a los cambios medioambientales ${ }^{(20)}$, aunque dichos autores reconocen que no fue posible asociar un genotipo kgp específico con severidad de la periodontitis. Por otra parte, las variantes de Kgp han sido clasificadas en 4 biotipos, de acuerdo a la heterogeneidad de los alelos del gen $\mathrm{kgp}$ en cepas de laboratorio de $P$. gingivalis, encontrando un biotipo (HG66) pobremente pigmentado ${ }^{(24)}$. Este hecho es relevante dado que la adquisición de hemina puede tener incidencia directa en la patogenicidad y sobrevida de este microorganismo(25), existiendo una asociación entre el gen kgp y posibles implicancias en la virulencia que presenta esta bacteria.

Los resultados presentados en este trabajo constituyen un primer reporte de la prevalencia de los genotipos rgpA y kgp en aislados de $P$. gingivalis obtenidos desde sujetos chilenos con periodontitis. A pesar del reducido número de individuos analizados en nuestro estudio, se observó una frecuencia de detección similar de genotipos rgpA y kgp a la descrita en la literatura. Cabe destacar que los aislados utilizados en dichas investigaciones fueron obtenidos de sujetos con periodontitis provenientes de distintas localizaciones geográficas (Alemania, Reino Unido, Suecia, Japón, Kenya, China y Estados Unidos) $)^{(19-21)}$
Otro aspecto a analizar es la detección de ambos genotipos kgp en los aislados provenientes de un mismo individuo, en contraste a lo obtenido por Beikler y cols. ${ }^{(20)}$, en donde no observaron colonización simultánea de perfiles $\mathrm{kgp}$ en un mismo paciente. Algunos estudios han establecido que un individuo con periodontitis porta un solo tipo clonal de $P$. gingivalis ${ }^{(26,27)}$. Sin embargo, otros autores utilizando distintas técnicas moleculares, han descrito que existe heterogeneidad clonal intra-individual para la colonización de $P$. gingivalis en pacientes con periodontitis ${ }^{(22,28,29)}$ lo cual reafirmaría lo encontrado en el presente estudio. En conclusión el análisis de los genotipos rgpA y kgp, mostró que los aislados de $P$. gingivalis no presentaron polimorfismos para el gen rgpA. En cambio, se observaron los dos perfiles descritos para el gen $k g p$, existiendo predominio del genotipo $\mathrm{kgp}-\mathrm{I}$. Sin embargo, para comprender de manera clara las implicancias de la mayor frecuencia detectada de este genotipo $(k g p-l)$, es necesario contar con más evidencia que evalúe si existen diferencias en su virulencia, analizar un mayor número de aislados y tratar de comprender su participación en la patogénesis de la periodontitis.

\section{CONFLICTOS DE INTERÉS}

Los autores declaran no tener conflictos de interés.

\section{REFERENCIAS BIBLIOGRÁFICAS}

1. Consensus report. Periodontal diseases: Pathogenesis and microbial factors. Ann Periodontol, 1996; 1: 926-932.

2. Socransky SS, Haffajee AD, Cugini MA, Smith C, Kent RL Jr. Microbial complexes in subgingival plaque. J Clin Periodontol, 1998; 25: 134-144.

3. Griffen AL, Becker MR, Lyons SR, Moeschberger ML, Leys EJ. Prevalence of Porphyromonas gingivalis and periodontal health status. J Clin Microbiol, 1998; 36: 3239-3242.

4. Silva N, Dutzan N, Hernandez M et al. Characterization of progressive periodontal lesions in chronic periodontitis patients: Levels of chemokines, cytokines, matrix metalloproteinase-13, periodontal pathogens and inflammatory cells. J Clin Periodontol, 2008; 35: 206-214.

5. Lamont RJ, Jenkinson HF. Life below the gum line: Pathogenic mechanisms of Porphyromonas gingivalis. Microbiol Mol Biol Rev, 1998; 62: 1244-1263.

6. Pike R, McGraw W, Potempa J, Travis J. Lysine-and arginine-specific proteinases from Porphyromonas gingivalis. Isolation, characterization, and evidence for the existence of complexes with hemagglutinins. J Biol Chem, 1994; 269: 406-411.

7. Curtis MA, Aduse-Opoku J, Rangarajan M. Cysteine proteases of Porphyromonas gingivalis. Critical reviews in oral biology and medicine: An official publication of the American Association of Oral Biologists, 2001: 12: 192-216.

8. Potempa J, Banbula A, Travis J. Role of bacterial proteinases in matrix destruction and modulation of host responses. Periodontol 2000, 2000; 24: 153-192.

9. Kantyka $T$, Latendorf $T$, Wiedow $O$ et al. Elafin is specifically inactivated by RgpB from Porphyromonas gingivalis by distinct proteolytic cleavage. Biological Chemistry, 2009; 390: 1313-1320.

10. Lewis JP. Metal uptake in host-pathogen interactions: Role of iron in Porphyromonas gingivalis interactions with host organisms. Periodontol 2000, 2010; 52: 94-116.

11. Chen T, Nakayama K, Belliveau L, Duncan MJ. Porphyromonas gingivalis gingipains and adhesion to epithelial cells. Infect Immun, 2001; 69: 3048-3056.

12. Kontani $\mathrm{M}$, Ono H, Shibata $\mathrm{H}$ et al. Cysteine protease of Porphyromonas gingivalis 381 enhances binding of fimbriae to cultured human fibroblasts and matrix proteins. Infect Immun, 1996; 64: 756-762.

13. Kinane JA, Benakanakere MR, Zhao J, Hosur KB, Kinane DF. Porphyromonas gingivalis influences actin degradation within epithelial cells during invasion and apoptosis. Cell Microbiol, 2012; 14: 1085-1096.

14. Carlisle MD, Srikantha RN, Brogden KA. Degradation of human alpha-and betadefensins by culture supernatants of Porphyromonas gingivalis strain 381. Journal of Innate Immunity, 2009; 1: 118-122.

15. Schenkein HA, Fletcher HM, Bodnar M, Macrina FL. Increased opsonization of a prtH-defective mutant of Porphyromonas gingivalis W83 is caused by reduced degradation of complement-derived opsonins. J Immunol, 1995; 154: 5331-5337. 16. Kitamura Y, Matono S, Aida Y, Hirofuji T, Maeda K. Gingipains in the culture supernatant of Porphyromonas gingivalis cleave CD4 and CD8 on human T cells. $J$ Periodontal Res, 2002; 37: 464-468.
17. Lourbakos A, Potempa J, Travis $\mathrm{J}$ et al. Arginine-specific protease from Porphyromonas gingivalis activates protease-activated receptors on human oral epithelial cells and induces interleukin-6 secretion. Infect Immun, 2001; 69: 5121 5130

18. Oido-Mori $\mathrm{M}$, Rezzonico $\mathrm{R}$, Wang $\mathrm{PL}$ et al. Porphyromonas gingivalis gingipain- $R$ enhances interleukin-8 but decreases gamma interferon-inducible protein 10 production by human gingival fibroblasts in response to T-cell contact. Infect Immun, 2001; 69: 4493-4501.

19. Allaker RP, Aduse-Opoku J, Batten JE, Curtis MA. Natural variation within the principal arginine-specific protease gene, prpR1, of Porphyromonas gingivalis. Oral Microbiol Immunol, 1997; 12: 298-302.

20. Beikler T, Peters U, Ehmke B, Flemmig TF. Sequence analysis of kgp in Porphyromonas gingivalis isolates from periodontitis patients. Oral Microbiol Immunol, 2003; 18: 393-397.

21. Yoshino T, Laine ML, van Winkelhoff AJ, Dahlen G. Genotype variation and capsular serotypes of Porphyromonas gingivalis from chronic periodontitis and periodontal abscesses. FEMS Microbiology Letters, 2007; 270: 75-81.

22. Abusleme L, Pozo P, Silva N. Genotipificación de Porphyromonas gingivalis en pacientes con periodontitis. Rev Clin Periodoncia Implantol Rehabil Oral, 2009; 2(2): 54-58.

23. Beikler T, Peters U, Prior K, Ehmke B, Flemmig TF. Sequence variations in rgpA and $r g p B$ of Porphyromonas gingivalis in periodontitis. J Periodontal Res, 2005 40: $193-198$

24. Nadkarni MA, Nguyen KA, Chapple CC, DeCarlo AA, Jacques NA, Hunter N Distribution of Porphyromonas gingivalis biotypes defined by alleles of the kgp (Lysgingipain) gene. J Clin Microbiol, 2004; 42: 3873-3876.

25. Shi Y, Ratnayake DB, Okamoto K, Abe N, Yamamoto K, Nakayama K Genetic analyses of proteolysis, hemoglobin binding, and hemagglutination of Porphyromonas gingivalis. Construction of mutants with a combination of rgpA, rgpB, kgp, and hagA. J Biol Chem, 1999; 274: 17955-17960.

26. Tribble GD, Lamont GJ, Progulske-Fox A, Lamont RJ. Conjugal transfer of chromosomal DNA contributes to genetic variation in the oral pathogen Porphyromonas gingivalis. J Bacteriol, 2007; 189: 6382-6388.

27. Park OJ, Min KM, Choe SJ, Choi BK, Kim KK. Use of insertion sequence element IS1126 in a genotyping and transmission study of Porphyromonas gingivalis. J Clin Microbiol, 2004; 42: 535-541.

28. Loos BG, Mayrand D, Genco RJ, Dickinson DP. Genetic heterogeneity of Porphyromonas (Bacteroides) gingivalis by genomic DNA fingerprinting. J Dent Res, 1990; 69: 1488-1493.

29. van Winkelhoff AJ, Rijnsburger MC, van der Velden U. Clonal stability of Porphyromonas gingivalis in untreated periodontitis. J Clin Periodontol, 2008; 35 674-679. 